IUCrJ

ISSN 2052-2525

BIOLOGY|MEDICINE

Received 31 January 2020

Accepted 3 June 2020

Edited by K. Moffat, University of Chicago, USA

₹ Present address: Laboratory of Biomolecular Research, Paul Scherrer Institute, CH-5232 Villigen PSI, Switzerland.

$\S$ Present address: European Molecular Biology Laboratory (EMBL) c/o DESY, D-22607

Hamburg, Germany.

Keywords: room-temperature oscillation X-ray crystallography; time-resolved crystallography; serial crystallography; plant photoreceptors.

PDB references: Structure of the light state of the LOV2 domain of phototropin-2 from

Arabidopsis thaliana, 6s46; Structure of the dark state of the LOV2 domain of phototropin-2 from Arabidopsis thaliana, 6s45

Supporting information: this article has supporting information at www.iucrj.org

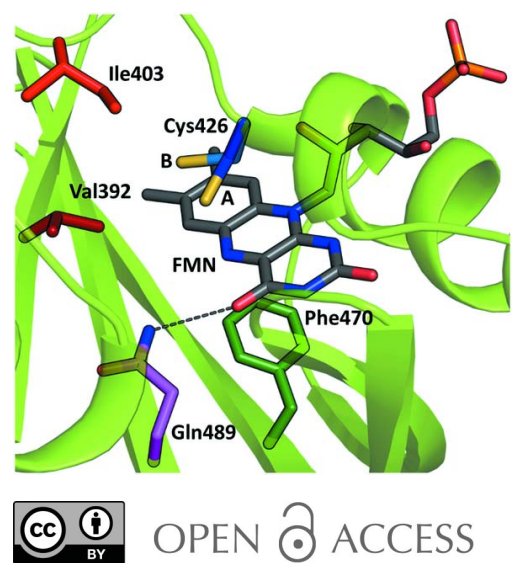

\section{Millisecond time-resolved serial oscillation crystallography of a blue-light photoreceptor at a synchrotron}

\author{
Sylvain Aumonier, ${ }^{\mathrm{a}, \mathrm{b}}$ Gianluca Santoni, ${ }^{\mathrm{a}}$ Guillaume Gotthard, ${ }^{\mathrm{a}}$ ‡ David von \\ Stetten, ${ }^{\mathrm{a}} \S$ Gordon A. Leonard ${ }^{\mathrm{a}}$ and Antoine Royant ${ }^{\mathrm{a}, \mathrm{b}_{*}}$
}

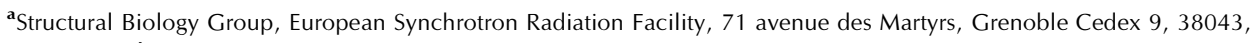
France, and ${ }^{\mathbf{b}}$ Institut de Biologie Structurale (IBS), Université Grenoble Alpes, CEA, CNRS, 71 avenue des Martyrs, Grenoble Cedex 9, 38044, France. *Correspondence e-mail: antoine.royant@ibs.fr

The recent development of serial crystallography has popularized time-resolved crystallography as a technique to determine the structure of protein-reaction intermediate states. However, most approaches rely on the availability of thousands to millions of microcrystals. A method is reported here, using monochromatic synchrotron radiation, for the room-temperature collection, processing and merging of $\mathrm{X}$-ray oscillation diffraction data from $<100$ samples in order to observe the build up of a photoreaction intermediate species. Using this method, we monitored with a time resolution of $63 \mathrm{~ms}$ how the population of a blue-light photoreceptor domain in a crystal progressively photoconverts from the dark to the light state. The series of resulting snapshots allows us to visualize in detail the gradual rearrangement of both the protein and chromophore during this process.

\section{Introduction}

Synchrotron-based X-ray crystallography has been largely responsible for the explosion in macromolecular crystallography, which has occurred since the early 2000s (http:// biosync.sbkb.org) and during which the overwhelming fraction of the structural information was obtained by exploiting crystals in which the conformation and biological state of the system under study is static, whether held at cryogenic or room temperature. However, gaining insight into the molecular mechanism of a protein greatly benefits from watching it in action [i.e. while it is performing its function (Hajdu et al., 2000; Bourgeois \& Royant, 2005; Šrajer \& Schmidt, 2017)] and, for this, time-resolved crystallography (TRX) experiments are necessary. Room-temperature Laue crystallography pioneered TRX at synchrotrons, providing a time resolution down to the $100 \mathrm{ps}$ level (Schotte et al., 2003). However, the number of systems to which the method could be applied is limited. Thus, although the technique produced some impressive successes (Wöhri et al., 2010; Schotte et al., 2012, 2003), TRX gradually fell out of favour. Recently, the development of serial crystallography (SX) (Chapman et al., 2011), coupled with advances in sample delivery (Grünbein \& Kovacs, 2019) and X-ray detectors (Broennimann et al., 2006; Casanas et al., 2016), has resulted in a renewed interest in TRX, first at free-electron lasers (TR-SFX, time-resolved serial femtosecond crystallography) (Barends et al., 2015; Pande et al., 2016; Nango et al., 2016; Kern et al., 2018), then at synchrotron sources (TR-SSX, time-resolved serial synchrotron crystallography) (Schulz et al., 2018; Mehrabi, Schulz, Dsouza et al., 2019; Mehrabi, Schulz, Agthe et al., 2019). 
(a)

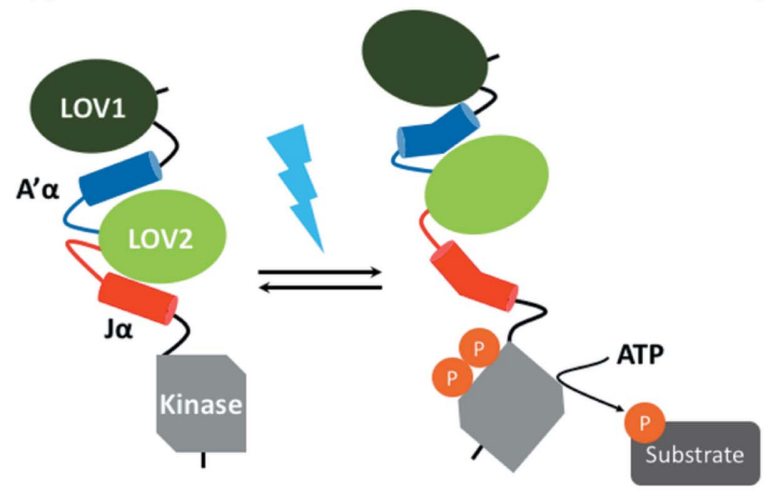

b)

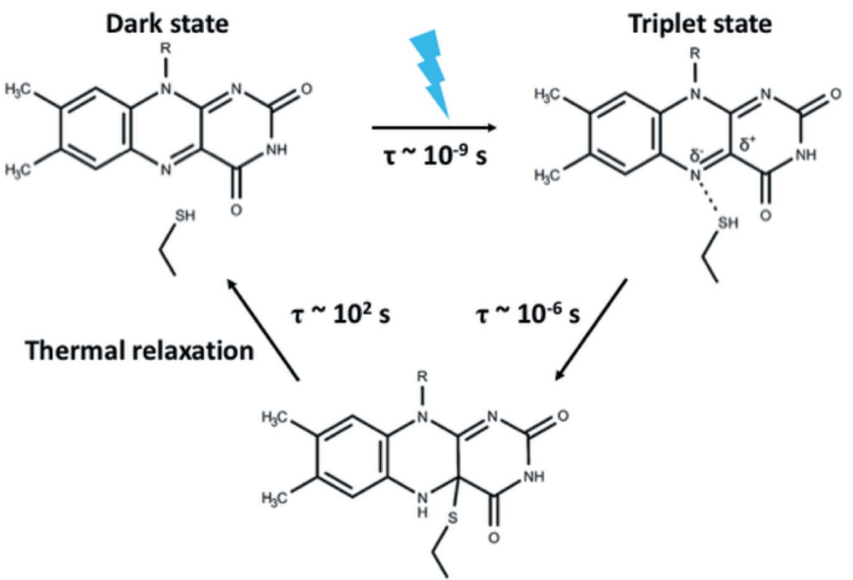

Light state (c)

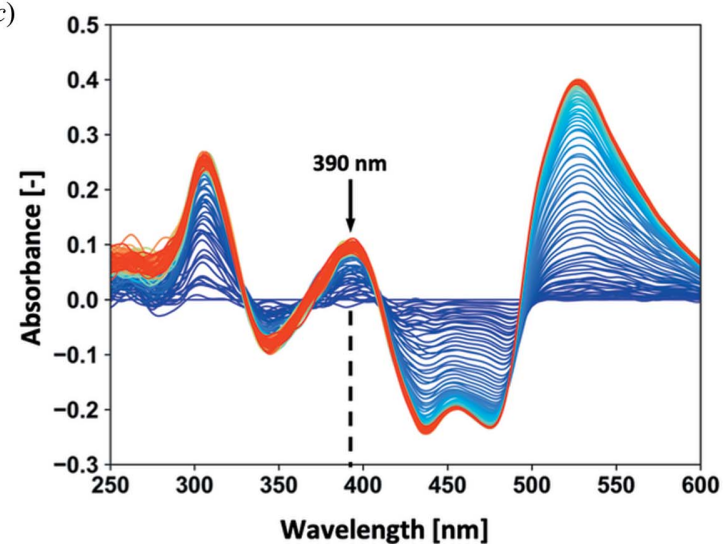

$(d)$

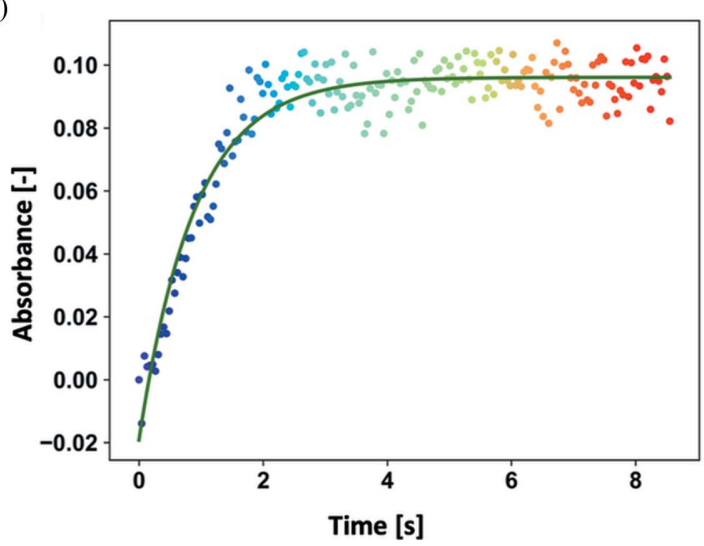

Figure 1

Photoactivation of the LOV2 domain of Phot2 from A. thaliana. (a) Schematic of Phot2 domain reorganization upon blue-light illumination (adapted from the work of Okajima et al., 2014). (b) The photocycle of AtPhot2LOV2. (c) Time-resolved series of UV-Vis absorption difference spectra of a AtPhot2LOV2 crystal under $3.2 \mu \mathrm{W}$ irradiation of a $470 \mathrm{~nm}$ LED, recorded at $23 \mathrm{~Hz}$ [colour range: violet $(t=0 \mathrm{~s})$ to red $(t=9 \mathrm{~s})$ ]. The absorption maximum of the light state is indicated with an arrow. We note that the apparent increase in absorbance above $500 \mathrm{~nm}$ is caused by the decrease in darkstate blue-light-induced fluorescence. (d) Evolution of the optical density at $\lambda=390 \mathrm{~nm}$, which is characteristic of the AtPhot2LOV2 light-state build up (rise time $\tau=0.891 \mathrm{~s}$ ).

However, both TR-SFX and TR-SSX techniques are, in the main, currently based on the recording of still diffraction images from many thousands of crystals for each time point, and their requirement in sample quantity is thus very high.

As an alternative to TR-SSX, we have developed a method, which we called time-resolved serial oscillation crystallography (TR-SOX), that exploits diffraction data collected from only tens to hundreds of crystals and has the potential to produce millisecond time-resolution movies of biomolecular processes occurring in crystals. TR-SOX was used to probe the build up of the light state in the photocycle of the LOV2 domain of Phot2 (phototropin-2) from Arabidopsis thaliana (AtPhot2LOV2) with a time resolution of $63 \mathrm{~ms}$. Phot2 is a high-intensity blue-light photoreceptor mediating phototropism in higher plants (Christie, 2007). The protein comprises two successive light-oxygen-voltage (LOV) domains (LOV1 and LOV2) separated by the $\alpha$-helix $\mathrm{A}^{\prime} \alpha$, and a kinase domain separated from LOV2 by the $\alpha$-helix J $\alpha$ [Fig. $1(a)$ ]. Through their connection to the LOV1 and kinase domains, both $\alpha$-helices can play a role in photoreceptor dimerization, prolongation of the photoreceptor signalling state duration or signal transduction (Halavaty \& Moffat, 2013; Okajima et al., 2014). In particular, J $\alpha$ unfolds upon bluelight irradiation, triggering kinase activity (Harper et al., 2003). The photoactivation of LOV domains has been intensively studied by various biophysical methods including fast spectroscopic techniques (Swartz et al., 2001; Kottke et al., 2003; Kennis et al., 2003). The dark state of AtPhot2LOV2 absorbs light maximally at $\lambda=447 \mathrm{~nm}$ via its flavin mononucleotide (FMN) chromophore. Upon photon absorption, a triplet state absorbing at $\lambda=660 \mathrm{~nm}$ forms in nanoseconds, which then converts on the microsecond time scale to the light state, a covalent flavin-cysteinyl adduct absorbing at $\lambda=390 \mathrm{~nm}$ [Fig. $1(b)]$. Finally, the system relaxes back to the dark state on the 100 s level.

\section{Methods}

\subsection{Protein expression and purification}

The gene coding for the Phot2 LOV2 domain from A. thaliana (AtPhot2LOV2) with a C-terminal hexahistidine 
tag was synthetized (Genecust, Ellange, Luxemburg) and inserted in a pBad vector using BamHI/PmeI cloning sites. The vector was transformed into the Escherichia coli host strain BL21 (Invitrogen) and grown on ampicillin $\left(100 \mu \mathrm{g} \mathrm{ml}^{-1}\right)$ selective agar plates. Cells were grown in 2YT medium supplemented with ampicillin $\left(100 \mu \mathrm{g} \mathrm{ml}^{-1}\right)$ at $37^{\circ} \mathrm{C}$ until an optical density of 0.6 at $600 \mathrm{~nm}$ was reached. Protein expression was then induced with $0.02 \%$ arabinose and cells were grown overnight at $17^{\circ} \mathrm{C}$. Cells were harvested and centrifuged at $4000 \mathrm{~g}$ for $20 \mathrm{~min}$ at $4{ }^{\circ} \mathrm{C}$. Pellets were resuspended in $25 \mathrm{ml}$ of lysis buffer [ $50 \mathrm{~m} M$ Tris $\mathrm{pH} 8.0,300 \mathrm{mM}$ $\mathrm{NaCl}, 10 \mathrm{mM}$ imidazole, $0.25 \mathrm{mg} \mathrm{ml}^{-1}$ lysozyme, $400 \mu \mathrm{g} \mathrm{ml}^{-1}$ DNAse I, $20 \mathrm{mM} \mathrm{MgSO}_{4}, 1$ tablet of the EDTA-free protease inhibitor cocktail cOmplete (Roche, Basel, Switzerland)] per litre of centrifuged medium and frozen at $-80^{\circ} \mathrm{C}$. Thawed pellets were sonicated and cell debris was centrifuged at $15000 \mathrm{~g}$, for $45 \mathrm{~min}$ at $4^{\circ} \mathrm{C}$. The protein was purified from the clarified lysate using a nickel affinity column (His-Trap HP $5 \mathrm{ml}$, GE HealthCare) and eluted against an imidazole gradient $(50 \mathrm{~m} M$ Tris $\mathrm{pH} 8.0,300 \mathrm{~m} M \mathrm{NaCl}, 10-500 \mathrm{~m} M$ imidazole). A second purification step consisted of sizeexclusion chromatography (Superdex 75 10/300 GL, GE HealthCare) in a $20 \mathrm{~m} M$ Tris $\mathrm{pH} 8.0$ buffer, after which the purified protein was concentrated to $8 \mathrm{mg} \mathrm{ml}^{-1}$.

\subsection{Protein crystallization}

Prior to crystallization, AtPhot2LOV2 aliquots were submitted to limited proteolysis using $0.5 \mu \mathrm{g} \mu \mathrm{l}^{-1}$ trypsin, which had been found to be crucial in controlling crystal quality. A first batch of crystals were produced by the hangingdrop vapour diffusion method ( $2 \mu \mathrm{l}$ drops of $1: 1$, mother liquor:protein ratio) in a condition consisting of $12-17 \%$ PEG8000, $200 \mathrm{~m} M$ calcium acetate in $100 \mathrm{~m} M$ MES buffer $\mathrm{pH}$ 6.0. This resulted in square-shaped crystals of heterogeneous size, which were then used for microseeding with a 1:100 dilution of the seed solution with the protein solution. The resulting crystals are of a much more homogeneous size of $50 \times 50 \times 50 \mu \mathrm{m}$.

\subsection{In crystallo UV-visible absorption spectroscopy}

UV-vis absorption spectra of AtPhot2LOV2 were obtained at the $i c \mathrm{OS}$ Laboratory at the European Synchrotron Radiation Facility (ESRF) (von Stetten et al., 2015). The reference light is provided by a DH2000-BAL lamp (Ocean Optics, Largo, Florida) and connected to the setup by a $200 \mu \mathrm{m}$ optical fibre. The transmitted light is connected to a gratingbased QE65Pro spectrophotometer (Ocean Optics, Largo, Florida) via a $400 \mu \mathrm{m}$ fibre to the output detector. The actinic light is output by a light-emitting diode (LED) of peak wavelength $\lambda=470 \mathrm{~nm}$ (Model M470F3, Thorlabs, Newton, New Jersey) connected to the setup via a $1000 \mu \mathrm{m}$ fibre. The tuneable power of the LED was checked with an energy meter (Nova II, Ophir, Jerusalem, Israel) in the range 0-27.5 $\mathrm{mW}$. The resulting power on the crystal was thus varied between 0 and $3.2 \mu \mathrm{W}$.

\subsection{Diffraction data collection and sorting}

All diffraction data were collected using ESRF beamline ID30A-3 (Theveneau et al., 2013; von Stetten et al., 2020). Each crystal was maintained at room temperature using a humidity controller (HC1, Arinax, Voreppe, France) with a relative humidity set at $99.65 \%$ [value determined from the composition of the mother liquor (Wheeler et al., 2012)]. The LED output was collimated at the entrance of a $5 \times$ magnifying objective positioned $75 \mathrm{~mm}$ from the sample position so as to deliver $3.5 \mu \mathrm{W}$ to the crystal (see Figs. S1 and S2 in the Supporting information). The limited illumination power ensures a gradual slow photo-conversion of the whole crystal from the top right corner (point of the illumination) to the bottom left corner (see Video S1 in the Supporting information).

Each crystal was manually mounted on the MD2 microdiffractometer (Arinax, Voreppe, France) with the top right corner of the crystal centred, to our best efforts, in the $15 \mu \mathrm{m}$ diameter X-ray beam. Each data collection comprised 1000 images of $0.5^{\circ}$ oscillation collected with an exposure time of $4.2 \mathrm{~ms}$. For each data set the first 990 images were binned into $66 \times 15$-image sub-data sets, corresponding to 66 time points. In order to prevent significant resolution loss during data collection caused by global radiation damage, the X-ray flux was limited to between $4.4 \times 10^{10}$ and $5.7 \times 10^{10}$ photons s $^{-1}$, corresponding to an absorbed dose of between 115 and $162 \mathrm{kGy}$ per full data collection. We performed 88 such data collections to monitor the build up of the light state. The LED was triggered by the rising edge of the TTL (transistortransistor logic) signal used to trigger the EIGER $\mathrm{X} 4 \mathrm{M}$ detector, which signals the start of the data collection synchronized with X-ray shutter opening and goniometer rotation. In order to generate the reference dark-state structure, we repeated a similar experiment on 32 different crystals without LED illumination.

\subsection{Diffraction data set processing}

All of the crystals belonged to the space group $P 4_{3} 2{ }_{1} 2$. For each of the 88 light-state data sets, and in anticipation of the situation where all 15-image sub-wedges would come from independent crystals thus allowing each crystal to absorb a significantly higher X-ray dose, each 15 -image sub-wedge was separately integrated using $X D S$ (Kabsch, 2010). The 66 subwedges of each of the 88 data sets corresponding to the 66 time points were then subjected to analysis using ccCluster (Santoni et al., 2017), which clusters isomorphous sub-data sets and eliminates outliers. Based on the output of the program, suitable clusters of sub-wedges were carried forward to the next stage. Here, reflections were combined into a single file using POINTLESS (Evans, 2011) and symmetry-related reflections were then scaled and merged using AIMLESS (Evans \& Murshudov, 2013). Final resolution limits were assigned using a $\mathrm{CC}_{1 / 2}$ cutoff (Karplus \& Diederichs, 2012) of 0.70 in the highest-resolution shell with $\langle I / \sigma(I)\rangle$ greater than 1.0. Inclusion of clustering nodes with increasing distance cutoffs was iteratively tested until the diffraction resolution, as 
defined above, could be maximized. In order to obtain a highquality reference data set, the dark-state data set was composed by merging the images of the best cluster (composed of five data sets) obtained from the 32 data sets (restricted to their first 500 images, as they provided a sufficient quantity of data) recorded in the absence of LED illumination.

2.6. Structure determination and refinement of the dark and light states

A model for the dark-state data set (time point $t<0 \mathrm{~ms}$ ) was constructed from a previously determined room-temperature structure of AtPhot2LOV2 (see the PDB entry 6qqj) (Gotthard et al., 2019). Structure refinement (including the four parameters $x, y, z$ and $B$ for each atom and group occupancy for each residue in alternate conformation) was performed using Phenix (Adams et al., 2010) with iterative inspection of electron-density maps in Coot (Emsley et al., 2010). This led to the identification of ten residues in alternative conformations A and B, including Cys 426 . The model consists of residues 388 to 492 , Glu388 being the second residue before the first strand and Gly492 being the fourth residue after the fifth and final strand of the LOV2 domain. There is an additional methionine at the $\mathrm{N}$ terminus, added for expression purposes, and ten residues of the linker forming a short five-residue helix at the $\mathrm{C}$ terminus.

A model for the light state was obtained using the data set at time point $t_{66}=4158 \mathrm{~ms}$. Initial maps were calculated by inserting the fixed dark-state structure determined at $t<0 \mathrm{~ms}$. Residual densities in $F_{\text {obs }}-F_{\text {calc }}$ and $2 F_{\text {obs }}-F_{\text {calc }}$ maps were investigated, and checked in maps at earlier time points 60 to 65 for consistency. A number of residues showed clear displacements and were modelled as alternate conformation C. In order to allow for the accommodation of significant movements of the main chain, residues flanking those in alternate conformation $\mathrm{C}$ were also modelled as such. Overall, six continuous stretches of the protein sequence were modelled in alternate conformations $\mathrm{A}$ and $\mathrm{C}$. The two key residues Cys426 and Phe 470 were modelled in three conformations: Cys426A, Cys426B and Phe470A in the dark state; and Cys426C, Phe470C and Phe470D in the light state. For consistency of occupancy refinement, the ten residues with a conformer B in those stretches have been limited to their A conformer in the fixed dark state. Once a satisfying atomic model of the light state had been obtained, occupancies for each of the six strands were refined. The structures of the dark state (time point $t_{0}=0 \mathrm{~ms}$ ) and light state (time point $t_{66}=$ $4158 \mathrm{~ms}$ ) of AtPhot2LOV2 have been deposited in the PDB under entries $6 s 45$ and $6 s 46$.

\subsection{Occupancy refinement for all time points}

Structure refinement of each time point between $t_{1}=63$ and $t_{65}=4095 \mathrm{~ms}$ was performed by inserting a combination of the dark and light states with occupancies $0.5 / 0.5$ for conformation $\mathrm{A}$ and $\mathrm{C}$, and $0.33 / 0.33 / 0.33$ for conformations $\mathrm{A} / \mathrm{B} / \mathrm{C}$ or
A/C/D. The only parameters that were subsequently refined were the occupancies of protein segments 1 to 6 .

\section{Results}

In order to observe the phenomenon on the millisecond to second time scale, we slowed down the rate of light-state build-up within a crystal of AtPhot2LOV2 using an attenuated $470 \mathrm{~nm}$ LED as actinic light. Depending on the level of attenuation, the limited number of photons photo-activate all protein molecules within the crystal in a few seconds (Video S1) to tens of seconds. The probed phenomenon is thus not the build up of the light state (which occurs in microseconds at the single-molecule level), but that of its population within the crystal. We used in crystallo UV-vis absorption spectroscopy at the IBS/ESRF icOS Laboratory (von Stetten et al., 2015) to quantify this phenomenon as a function of actinic light power. Absorption spectra were continuously recorded at a $23 \mathrm{~Hz}$ rate providing a $43 \mathrm{~ms}$ time resolution. Build-up rates were observed to be linearly correlated with LED power over a 50fold power range (Fig. S1), proving that light-state build up in the crystal is solely accounted for by the number of photons impinging on the crystal. We thus determined that a light power of $3.2 \mu \mathrm{W}$ at the crystal surface would result in a buildup time constant just below $1 \mathrm{~s}$ (891 ms) [Figs. 1(c) and 1(d)].

For TR-SOX experiments on the ESRF beamline ID30A-3, 88 randomly-oriented crystals of AtPhot2LOV2 were sequentially mounted in a wet air stream at ambient temperature, under low ambient light conditions with LED illumination synchronized with X-ray shutter opening, goniometer axis rotation and X-ray detector acquisition. The X-ray beam flux was attenuated such that the total absorbed dose for each data set was between 115 and $162 \mathrm{kGy}$, thus minimizing resolution loss and thio-ether bond reduction (Gotthard et al., 2019). For each crystal, 990 images of $0.5^{\circ}$ oscillation range were recorded at an acquisition rate of $250 \mathrm{~Hz}$. Each of the 88 resulting data sets was then split into 15-frame sub-wedges assigned to 66 different time periods $T_{1}$ (0-63 ms) to $T_{66}(4095-4158 \mathrm{~ms})$, which were ascribed to 66 time points from $t_{1}=63 \mathrm{~ms}$ to $t_{66}=4158 \mathrm{~ms}$ [Fig. 2(a)].

For each time point of the diffraction experiment, between 36 and 52 sub-wedges were successfully integrated and merged together using ccCluster (Santoni et al., 2017) [Figs. 2(b) and $2(c)$ ]. The clustering threshold $\mathrm{CC}_{\text {threshold }}$ was chosen such that the diffraction resolution of each final data set was maximized (see Methods). We thus obtained 66 complete data sets corresponding to time points $t_{1}$ to $t_{66}$ at a diffraction resolution starting at $2.45 \AA$ for $t_{1}$ and rapidly settling at $2.75 \AA$ after $t_{11}=$ $693 \mathrm{~ms}$ [see Table S1 in the Supporting information and Fig. $3(a)$ ]. In addition to the final data set resolution, two other data integration and reduction parameters were also observed to evolve with time in a similar manner. First, the value $\mathrm{CC}_{\text {threshold }}$ used to maximize resolution diffraction increases steadily from 0.39 at $t_{1}$ and stabilizes around 0.68 after $t_{24}=$ $1512 \mathrm{~ms}$ [Fig. 3(b)]; second, the cell parameter $a$ follows a comparable pattern by increasing from $40.73 \AA$ at $t_{1}$ to an average of $40.97 \AA$ after $t_{34}=2142 \mathrm{~ms}$, while the cell parameter 


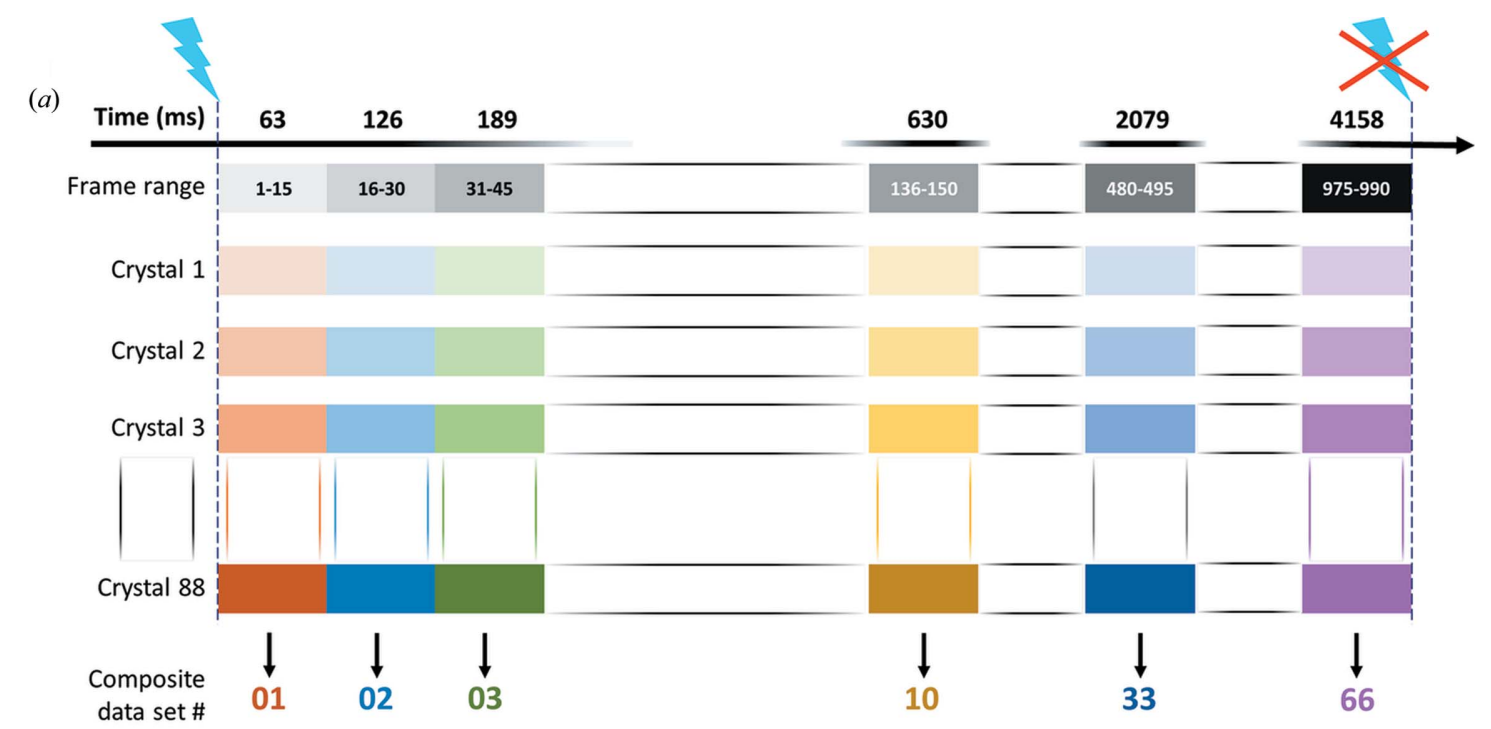

(b)
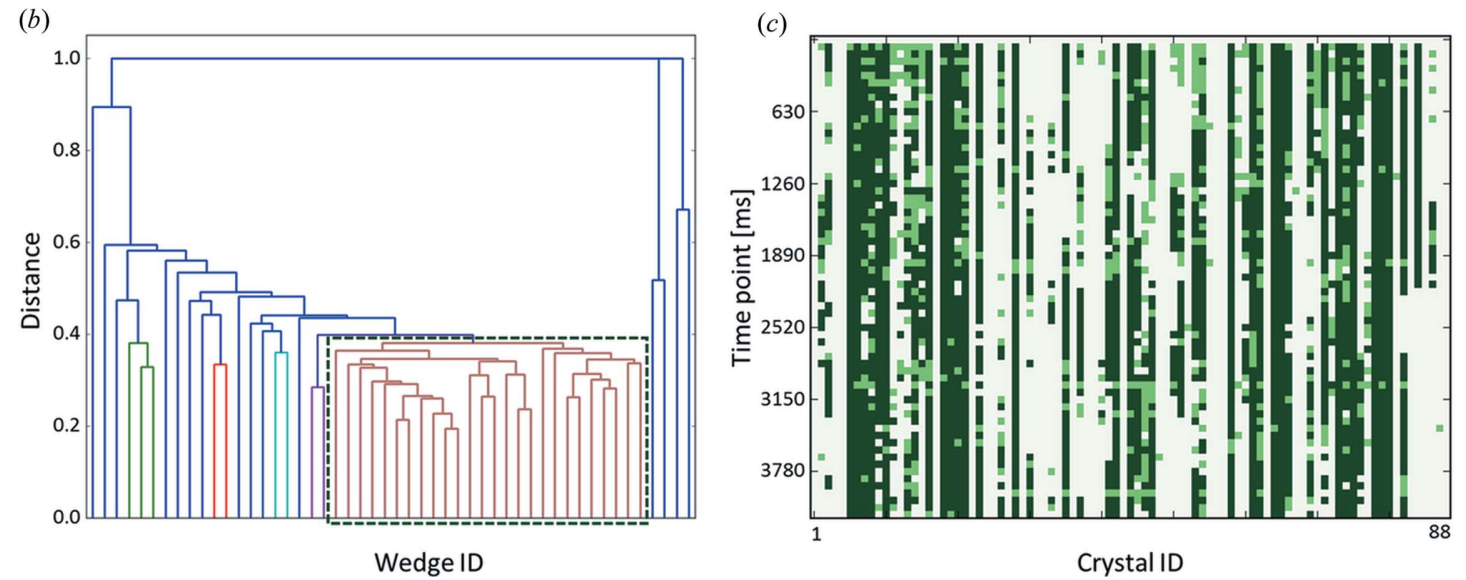

Figure 2

Data-collection strategy and data set composition. (a) Complete data sets were collected from 88 different crystals. For each crystal, the diffraction images were separated into 15 frame wedges corresponding to 66 time points (from 63 to $4158 \mathrm{~ms}$ ). (b) A dendrogram of wedge clustering for the initial time point $(t=0-63 \mathrm{~ms})$. The 26 wedges forming the cluster highlighted with a dark green rectangle group at a linkage distance of 0.39 were subsequently merged together to produce a $2.45 \AA$ resolution data set with $100 \%$ completeness and a multiplicity of 12.6 (Table S1). (c) A visualization of the data set wedges integrated (light and dark green) and of the wedges retained in the composite data sets corresponding to the 66 different time points (dark green only).

$c$ appears to remain constant within the noise [Figs. 3(c) and $3(d)]$. The fact that these three parameters settle to a plateau may indicate that their evolution is not primarily driven by $\mathrm{X}$-ray radiation damage but is potentially an effect of photoactivation of the protein leading to subtle changes in the unitcell parameters and crystal contacts and thus to a decrease, as a function of time, in the degree of isomorphism.

To determine the structure of the dark state of AtPhot2LOV2, representative of the time series before $t=$ $0 \mathrm{~ms}$, we carried out the same diffraction experiment, but without illumination, on a series of 32 crystals. The first 500 images of 15 data sets could be successfully integrated and were subjected to clustering with $c c$ Cluster which retained 5 sub-wedges using a $\mathrm{CC}_{\text {threshold }}$ of 0.43 and resulted in a complete highly redundant data set at $2.2 \AA$ resolution (Table S1). Refinement of the crystal-state structure of the dark state was then carried out using a previously determined structure of AtPhot2LOV2 (PDB entry 6qqj) (Gotthard et al., 2019) as a starting model. Most residues were modelled in a single conformation A, while ten were modelled with alternate conformations $\mathrm{A}$ and $\mathrm{B}$, including Cys 426 which forms a covalent bond with the FMN in the light state. The hydrophilic side of the FMN isoalloxazine ring is stabilized by two hydrogen bonds to Asn458 and one key hydrogen bond to Gln489 [Fig. 4(a)].

Modelling of the crystal structure of the light state of AtPhot2LOV2 [denoted C and D in Fig. 4(c)] was guided by inspection of $F_{\text {obs }}-F_{\text {calc }}$ and $2 F_{\text {obs }}-F_{\text {calc }}$ electron-density maps for the later time points $\left(t_{60}\right.$ to $\left.t_{66}\right)$ in our diffraction data series. Here, in addition to the formation of the covalent bond between Cys 426 and FMN and the rotation of the FMN moiety, several residues surrounding the chromophore show clear signs of side-chain translation (Gln489, Ile403, Val392), rotation and reorientation (Phe470) as well as displacement of 
(a)

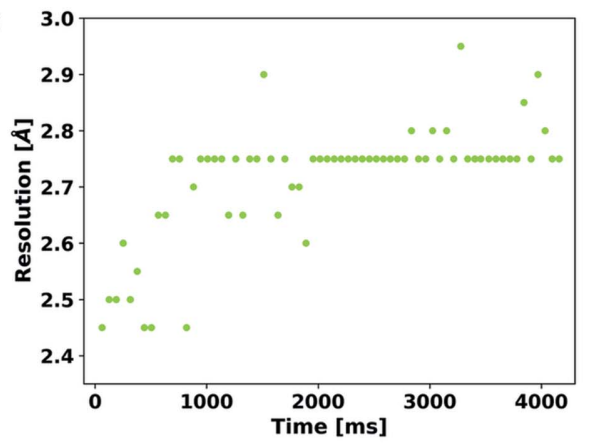

(c)

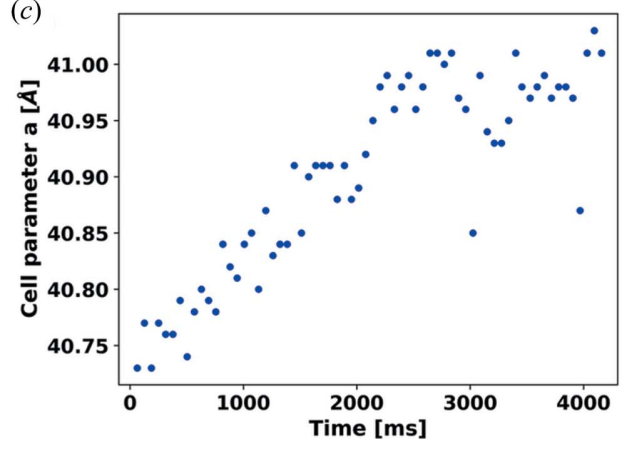

(b)

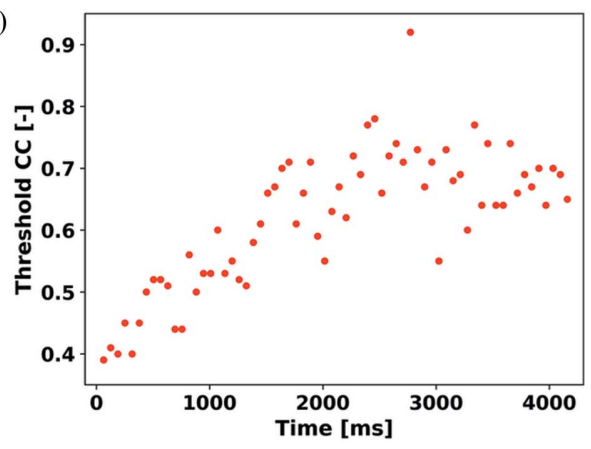

(d)

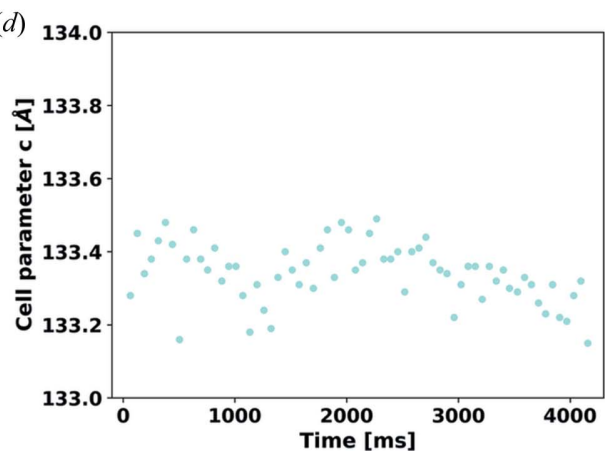

Figure 3

The evolution of various crystallographic data reduction parameters as a function of time. The evolution of $(a)$ diffraction resolution, $(b)$ distance threshold $\mathrm{CC}_{\text {threshold }}$ for the clustering of sub-data sets, $(c)$ unit-cell parameter $a$ and $(d)$ unit-cell parameter $c$.
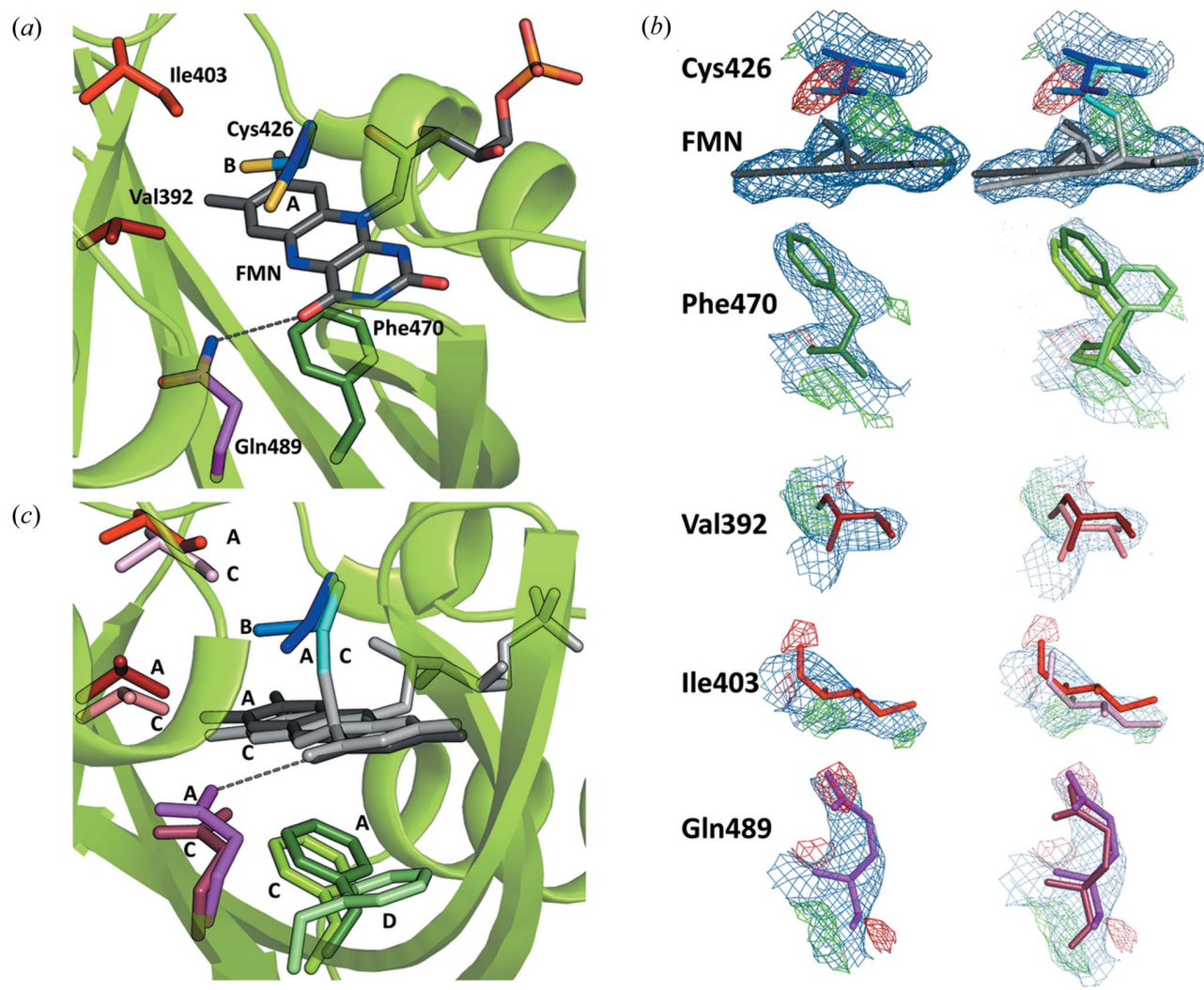

Figure 4

Model building of the structure of the light state of AtPhot2LOV2. (a) The structure of AtPhot2LOV2 in the dark state showing key residues surrounding the FMN chromophore. $(b) F_{\text {obs }}-F_{\text {calc }}$ (green, positive; red, negative) and $2 F_{\text {obs }}-F_{\text {calc }}$ electron-density maps, contoured at $2.5 \sigma$ and $1.0 \sigma$ levels, respectively, for all key residues and the FMN chromophore at time point 66 before modelling of the light state. The dark-state model (left) and both dark- and light-state models (right) are represented. (c) The structure of AtPhot2LOV2 in the light state (C and D conformers, light shade colours) superimposed on that of the dark state (A and B conformers, darker shade colours). 
(a)

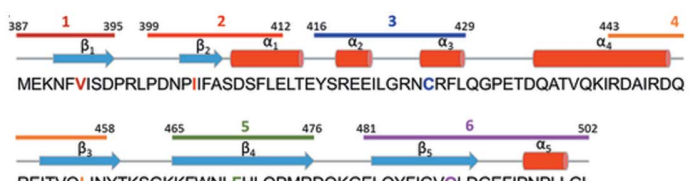

(b)
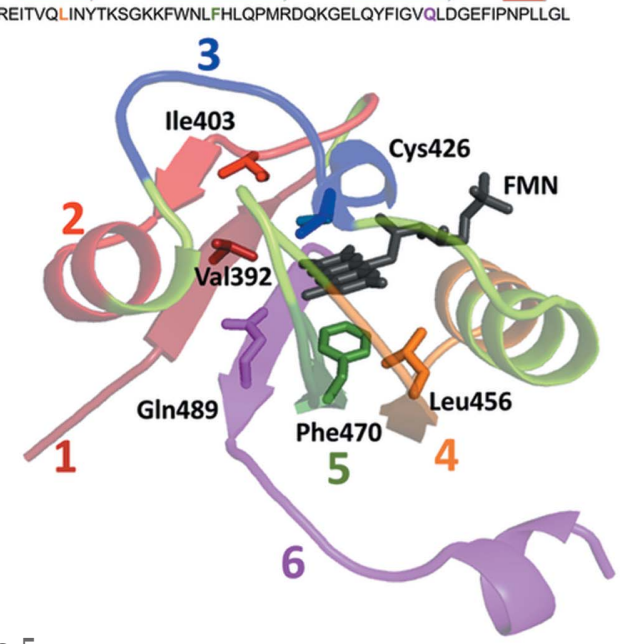

(c)

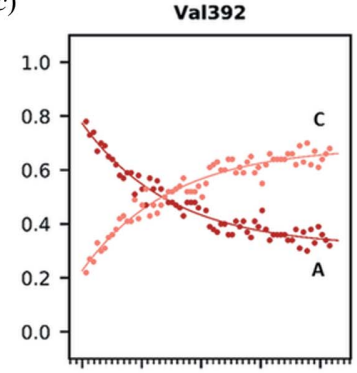

Phe470

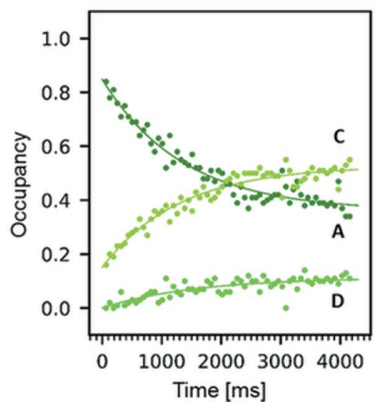

Ile403

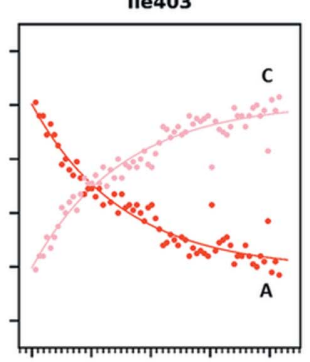

GIn489

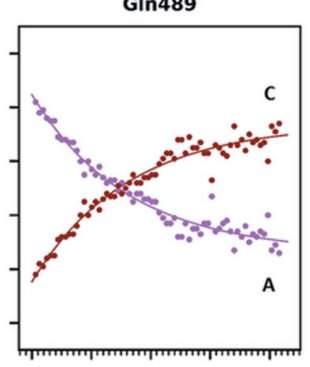

Cys426

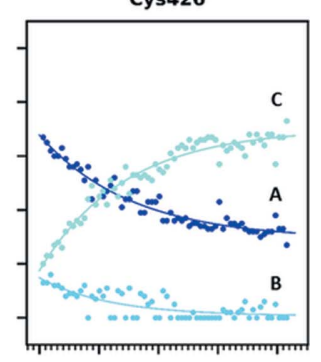

FMN

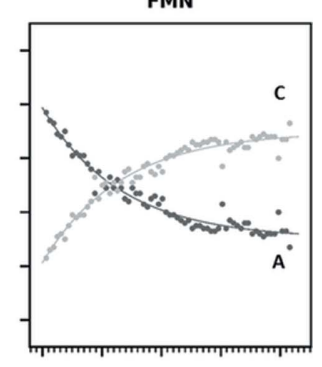

Figure 5

Time evolution of the relative occupancies of key residues of AtPhot2LOV2 upon blue-light illumination. $(a)$ Sequence and $(b)$ structure featuring key residues in colour, secondary structure elements ( $\alpha$-helices and $\beta$-strands) and protein segments 1 to 6 that move between the dark and the light state. (c) Occupancies of the different conformations (A, B: dark state; C, D: light state) are modelled as mono-exponential rises or decays. For every residue and the cofactor, occupancy and time scales are as shown for Phe470.

their main-chain atoms [Fig. 4(b)]. Previous structures of the light state of LOV domains, in particular those described in the work of Halavaty \& Moffat (2007), show a flipping of the head group of Gln489. However, in our study this flipping is not supported by atomic $B$-factor refinement, although this may be a result of a more dynamic disorder that we are unable to properly model. Phe470 is shown to adopt two new conformations $\mathrm{C}$ and $\mathrm{D}$, the latter of which is made possible by the displacement and reorientation of Leu456 (Fig. S3). We modelled continuous sequence stretches around those residues and refined a conformation $\mathrm{C}$ of the protein for each of the resulting segments 1 to 6 , all composed of rigid secondarystructure elements [Figs. 5(a), 5(b) and S4].

In order to follow the time course of the dark- to light-state conversion, we evaluated their respective structural contributions to each time point. Occupancies of the six segments were refined independently, while atomic coordinates and $B$ factors were fixed. The evolution of the respective occupancies of each of the prominent residues and cofactor are represented in Fig. 5(c). All decays (conformations A and B) and rises (conformations $\mathrm{C}$ and $\mathrm{D}$ ) could be modelled by a monoexponential behaviour, whose time constants are very similar, with an average value of $1445 \pm 135 \mathrm{~ms}$ (Table S2). This is in rather good agreement with the spectroscopy-derived rise time of $891 \mathrm{~ms}$ for light-state build up under similar experimental conditions [Fig. 1(d)]. Yet, there is a discrepancy between the maximum occupancy of the light state as observed by spectroscopy $(\sim 100 \%)$ and that observed by X-ray crystallography $(\sim 70 \%)$. We cannot exclude that a small fraction of the flavin-cysteinyl adduct may be reduced by X-rays but the probable explanation for the discrepancy must reside in our alternate-conformer refinement strategy, which may overestimate the proportion of the dark-state conformer at this resolution, whose structure is, overall, very close to that of the light state. Nevertheless, our results demonstrate that we have been able to visualize at the nearatomic scale the progressive conversion from the dark to the light state of AtPhot2LOV2 molecules within the crystal on an $\sim 60 \mathrm{~ms}$ time scale.

\section{Conclusions}

The evolution of $2 F_{\text {obs }}-F_{\text {calc }}$ electron-density maps and structural models obtained [Figs. 6(a) and 6(b) and Videos S2 and S3] allows an atomic description of the dark- to light-state conversion of $A t$ Phot2LOV2 to be determined. Absorption of a photon by FMN leads to the recruitment of both the A and B conformers of Cys 426 in segment 3 to establish a covalent bond between its $\mathrm{S}_{\gamma}$ atom and the $\mathrm{C}_{4 \mathrm{a}}$ atom of FMN. This results in an $\sim 6^{\circ}$ rotation of the chromophore isoalloxazine ring around the axis of its ribityl tail and in the disruption of a stabilizing hydrogen to Gln489. The disappearance of conformer B of Cys 426 added to the rotation of the isoalloxazine ring creates a void that is readily occupied by both Val392 and Ile 403 whose side chains translate by $0.6-1.1 \AA$ towards the FMN, displacing both protein segments 1 and 2 . The rotation of the chromophore pushes Phe 470 into two new conformers, thus displacing segment 5 . The minor conformer D of Phe 470 forces a rearrangement of Leu456, thus displacing segment 4. Finally, the change in electronic configuration of the FMN upon covalent bond formation induces the loss of the stabilizing hydrogen bond between the FMN and Gln489, whose main and side chains move away, displacing segment 6 . Segments 1 and 6 , constituting the $\mathrm{N}$ and $\mathrm{C}$ termini of the 


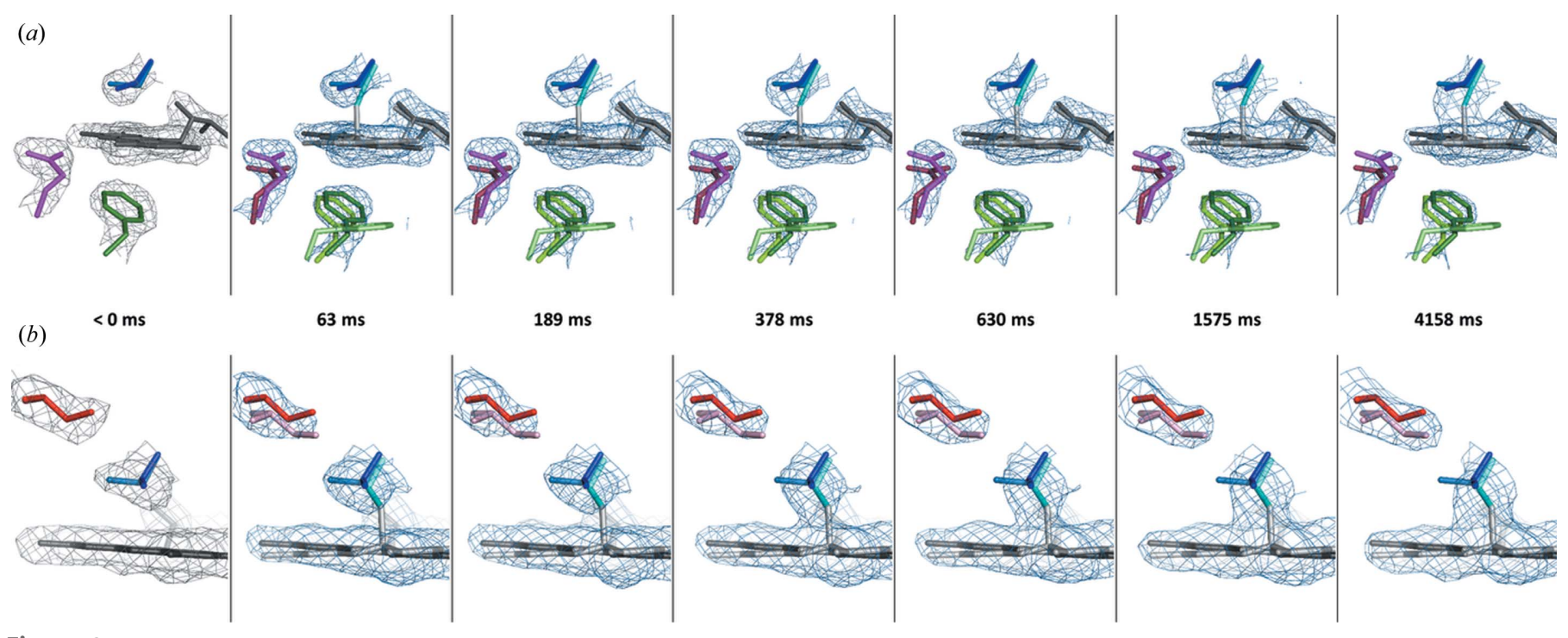

Figure 6

Snapshots of the molecular movie resulting from the TR-SOX experiment. (a) Snapshots at time points $0,1,3,6,10,25$ and 66 showing $2 F_{\text {obs }}-F_{\text {calc }}$ electron-density maps contoured at a $1.2 \sigma$ level superimposed on the structural models of the dark and light states highlighting covalent bond formation between Cys 426 and the FMN, disordering of the Gln489 side chain, and displacement of the Phe 470 side chain. $(b)$ A different view showing $2 F_{\text {obs }}-$ $F_{\text {calc }}$ electron-density maps contoured at a $0.7 \sigma$ level illustrating rotation of the isoalloxazine ring of the FMN, translation of Ile403 and progressive disappearance of the B conformation of Cys426.

protein, respectively, have already been shown to be involved in signal transduction through the modification of their interactions with the $\mathrm{A}^{\prime} \alpha$ and $\mathrm{J} \alpha$ helices (Halavaty \& Moffat, 2007). In particular, the structure of the LOV2-J $\alpha$ domain of Avena sativa (PDB entry 2v1a; Halavaty \& Moffat, 2007) reveals that the equivalent of segments 1,5 and 6 defined here form a groove accommodating the $\mathrm{J} \alpha$ helix and support the notion that their concerted displacement would destabilize this helix, which in turn would activate the kinase domain.

Here we have used room-temperature TR-SOX coupled with complementary in crystallo optical spectroscopy to follow, and provide structural insight into, the activation mechanism of a light-activated phototropin. One noteworthy advantage of this method, which is based on serial oscillation crystallography, is the significant reduction of sample material required compared with still-image-based crystallography. Obviously, our study has benefited from the high symmetry of the AtPhot2LOV2 crystal point group, and an $n$-fold increase in the number of crystals of another biological system which produces crystals with a point group of $n$-fold lower symmetry would have to be used in order to achieve the same time resolution, with the same diffraction resolution. We have used a conservative approach by limiting the absorbed dose to $\sim 170 \mathrm{kGy}$ for a complete data set on a single crystal, thus limiting the diffraction resolution of the reconstructed data sets. A straightforward extension of the method would be to record fewer sub-wedges from more individual crystals, thus allowing each crystal to absorb a higher dose, up to 66 times more, maximizing the resolution of resulting data sets. The method could be extended to non-photoactivable proteins by using microfluidics-based 'mix-and-diffuse' techniques, which would allow for $\mathrm{pH}$ jumps or the diffusion of substrates or ligands. Besides, we have shown that limiting the photon flux to the sample slows down the build up of the population of a sub-millisecond intermediate state from an initial state, which provides the opportunity to visualize the progressive conversion from one state to the other with a redundant set of information. In this study, the time resolution obtained was limited by a maximum goniometer rotation speed $\left(180^{\circ} \mathrm{s}^{-1}\right)$ and detector readout rate $(250 \mathrm{~Hz})$. Maximizing both of these aspects will probably lead to recording molecular movies on the sub-millisecond time scale. This will allow researchers to characterize large-scale structural changes that are often associated with photoreceptor or photoenzyme activation, providing an alternative to XFEL experiments for microsecond to millisecond protein dynamics studies.

\section{Acknowledgements}

The ESRF is acknowledged for access to beamlines and facilities for molecular biology via its in-house research programme. This work used the icOS Laboratory, which is a platform of the Grenoble Instruct-ERIC Centre (ISBG; UMS 3518 CNRS-CEA-UJF-EMBL) within the Grenoble Partnership for Structural Biology (PSB).

\section{Funding information}

SA acknowledges a PhD fellowship from the ESRF.

\section{References}

Adams, P. D., Afonine, P. V., Bunkóczi, G., Chen, V. B., Davis, I. W., Echols, N., Headd, J. J., Hung, L.-W., Kapral, G. J., GrosseKunstleve, R. W., McCoy, A. J., Moriarty, N. W., Oeffner, R., Read, R. J., Richardson, D. C., Richardson, J. S., Terwilliger, T. C. \& Zwart, P. H. (2010). Acta Cryst. D66, 213-221.

Barends, T. R. M., Foucar, L., Ardevol, A., Nass, K., Aquila, A., Botha, S., Doak, R. B., Falahati, K., Hartmann, E., Hilpert, M., 
Heinz, M., Hoffmann, M. C., Köfinger, J., Koglin, J. E., Kovacsova, G., Liang, M., Milathianaki, D., Lemke, H. T., Reinstein, J., Roome, C. M., Shoeman, R. L., Williams, G. J., Burghardt, I., Hummer, G., Boutet, S. \& Schlichting, I. (2015). Science, 350, 445-450.

Bourgeois, D. \& Royant, A. (2005). Curr. Opin. Struct. Biol. 15, 538-547.

Broennimann, Ch., Eikenberry, E. F., Henrich, B., Horisberger, R., Huelsen, G., Pohl, E., Schmitt, B., Schulze-Briese, C., Suzuki, M., Tomizaki, T., Toyokawa, H. \& Wagner, A. (2006). J. Synchrotron Rad. 13, 120-130.

Casanas, A., Warshamanage, R., Finke, A. D., Panepucci, E., Olieric, V., Nöll, A., Tampé, R., Brandstetter, S., Förster, A., Mueller, M., Schulze-Briese, C., Bunk, O. \& Wang, M. (2016). Acta Cryst. D72, 1036-1048.

Chapman, H. N., Fromme, P., Barty, A., White, T. A., Kirian, R. A., Aquila, A., Hunter, M. S., Schulz, J., DePonte, D. P., Weierstall, U., Doak, R. B., Maia, F. R. N. C., Martin, A. V., Schlichting, I., Lomb, L., Coppola, N., Shoeman, R. L., Epp, S. W., Hartmann, R., Rolles, D., Rudenko, A., Foucar, L., Kimmel, N., Weidenspointner, G., Holl, P., Liang, M., Barthelmess, M., Caleman, C., Boutet, S., Bogan, M. J., Krzywinski, J., Bostedt, C., Bajt, S., Gumprecht, L., Rudek, B., Erk, B., Schmidt, C., Hömke, A., Reich, C., Pietschner, D., Strüder, L., Hauser, G., Gorke, H., Ullrich, J., Herrmann, S., Schaller, G., Schopper, F., Soltau, H., Kühnel, K. U., Messerschmidt, M., Bozek, J. D., Hau-Riege, S. P., Frank, M., Hampton, C. Y., Sierra, R. G., Starodub, D., Williams, G. J., Hajdu, J., Timneanu, N., Seibert, M. M., Andreasson, J., Rocker, A., Jönsson, O., Svenda, M., Stern, S., Nass, K., Andritschke, R., Schröter, C. D., Krasniqi, F., Bott, M., Schmidt, K. E., Wang, X., Grotjohann, I., Holton, J. M., Barends, T. R. M., Neutze, R., Marchesini, S., Fromme, R., Schorb, S., Rupp, D., Adolph, M., Gorkhover, T., Andersson, I., Hirsemann, H., Potdevin, G., Graafsma, H., Nilsson, B. \& Spence, J. C. H. (2011). Nature, 470, 73-77.

Christie, J. M. (2007). Annu. Rev. Plant Biol. 58, 21-45.

Emsley, P., Lohkamp, B., Scott, W. G. \& Cowtan, K. (2010). Acta Cryst. D66, 486-501.

Evans, P. R. (2011). Acta Cryst. D67, 282-292.

Evans, P. R. \& Murshudov, G. N. (2013). Acta Cryst. D69, 1204-1214.

Gotthard, G., Aumonier, S., De Sanctis, D., Leonard, G., von Stetten, D. \& Royant, A. (2019). IUCrJ, 6, 665-680.

Grünbein, M. L. \& Nass Kovacs, G. (2019). Acta Cryst. D75, 178-191.

Hajdu, J., Wilmot, C. M., Neutze, R., Sjögren, T., Edman, K., Szöke, A. \& Wilmouth, R. C. (2000). Nat. Struct. Biol. 7, 1006-1012.

Halavaty, A. S. \& Moffat, K. (2007). Biochemistry, 46, 14001-14009. Halavaty, A. S. \& Moffat, K. (2013). Acta Cryst. F69, 1316-1321.

Harper, S. M., Neil, L. C. \& Gardner, K. H. (2003). Science, 301, 1541-1544.

Kabsch, W. (2010). Acta Cryst. D66, 125-132.

Karplus, P. A. \& Diederichs, K. (2012). Science, 336, 1030-1033.

Kennis, J. T. M., Crosson, S., Gauden, M., van Stokkum, I. H. M., Moffat, K. \& van Grondelle, R. (2003). Biochemistry, 42, 3385-3392.

Kern, J., Chatterjee, R., Young, I. D., Fuller, F. D., Lassalle, L., Ibrahim, M., Gul, S., Fransson, T., Brewster, A. S., Alonso-Mori, R., Hussein, R., Zhang, M., Douthit, L., de Lichtenberg, C., Cheah, M. H., Shevela, D., Wersig, J., Seuffert, I., Sokaras, D., Pastor, E., Weninger, C., Kroll, T., Sierra, R. G., Aller, P., Butryn, A., Orville, A. M., Liang, M., Batyuk, A., Koglin, J. E., Carbajo, S., Boutet, S., Moriarty, N. W., Holton, J. M., Dobbek, H., Adams, P. D., Bergmann, U., Sauter, N. K., Zouni, A., Messinger, J., Yano, J. \& Yachandra, V. K. (2018). Nature, 563, 421-425.

Kottke, T., Heberle, J., Hehn, D., Dick, B. \& Hegemann, P. (2003). Biophys. J. 84, 1192-1201.

Mehrabi, P., Schulz, E. C., Agthe, M., Horrell, S., Bourenkov, G., von Stetten, D., Leimkohl, J.-P., Schikora, H., Schneider, T. R., Pearson,
A. R., Tellkamp, F. \& Miller, R. J. D. (2019). Nat. Methods, 16, 979-982.

Mehrabi, P., Schulz, E. C., Dsouza, R., Müller-Werkmeister, H. M., Tellkamp, F., Miller, R. J. D. \& Pai, E. F. (2019). Science, 365, 1167-1170.

Nango, E., Royant, A., Kubo, M., Nakane, T., Wickstrand, C., Kimura, T., Tanaka, T., Tono, K., Song, C., Tanaka, R., Arima, T., Yamashita, A., Kobayashi, J., Hosaka, T., Mizohata, E., Nogly, P., Sugahara, M., Nam, D., Nomura, T., Shimamura, T., Im, D., Fujiwara, T., Yamanaka, Y., Jeon, B., Nishizawa, T., Oda, K., Fukuda, M., Andersson, R., Båth, P., Dods, R., Davidsson, J., Matsuoka, S., Kawatake, S., Murata, M., Nureki, O., Owada, S., Kameshima, T., Hatsui, T., Joti, Y., Schertler, G., Yabashi, M., Bondar, A.-N., Standfuss, J., Neutze, R. \& Iwata, S. (2016). Science, 354, 1552-1557. Okajima, K., Aihara, Y., Takayama, Y., Nakajima, M., Kashojiya, S., Hikima, T., Oroguchi, T., Kobayashi, A., Sekiguchi, Y., Yamamoto, M., Suzuki, T., Nagatani, A., Nakasako, M. \& Tokutomi, S. (2014). J. Biol. Chem. 289, 413-422.

Pande, K., Hutchison, C. D. M., Groenhof, G., Aquila, A., Robinson, J. S., Tenboer, J., Basu, S., Boutet, S., DePonte, D. P., Liang, M., White, T. A., Zatsepin, N. A., Yefanov, O., Morozov, D., Oberthuer, D., Gati, C., Subramanian, G., James, D., Zhao, Y., Koralek, J., Brayshaw, J., Kupitz, C., Conrad, C., Roy-Chowdhury, S., Coe, J. D., Metz, M., Xavier, P. L., Grant, T. D., Koglin, J. E., Ketawala, G., Fromme, R., rajer, V., Henning, R., Spence, J. C., Ourmazd, A., Schwander, P., Weierstall, U., Frank, M., Fromme, P., Barty, A., Chapman, H. N., Moffat, K., van Thor, J. J. \& Schmidt, M. (2016). Science, 352, 725-729.

Santoni, G., Zander, U., Mueller-Dieckmann, C., Leonard, G. \& Popov, A. (2017). J. Appl. Cryst. 50, 1844-1851.

Schotte, F., Cho, H. S., Kaila, V. R. I., Kamikubo, H., Dashdorj, N., Henry, E. R., Graber, T. J., Henning, R., Wulff, M., Hummer, G., Kataoka, M. \& Anfinrud, P. A. (2012). Proc. Natl Acad. Sci. USA, 109, 19256-19261.

Schotte, F., Lim, M., Jackson, T. A., Smirnov, A. V., Soman, J., Olson, J. S., Phillips, G. N., Wulff, M. \& Anfinrud, P. (2003). Science, 300, 1944-1947.

Schulz, E. C., Mehrabi, P., Müller-Werkmeister, H. M., Tellkamp, F., Jha, A., Stuart, W., Persch, E., De Gasparo, R., Diederich, F., Pai, E. F. \& Miller, R. J. D. (2018). Nat. Methods, 15, 901-904.

Šrajer, V. \& Schmidt, M. (2017). J. Phys. D Appl. Phys. 50, 373001.

Stetten, D. von, Carpentier, P., Flot, D., Beteva, A., Caserotto, H., Dobias, F., Guijarro, M., Giraud, T., Lentini, M., McSweeney, S., Royant, A., Petitdemange, S., Sinoir, J., Surr, J., Svensson, O., Theveneau, P., Leonard, G. A. \& Mueller-Dieckmann, C. (2020). J. Synchrotron Rad. 27, 844-851.

Stetten, D. von, Giraud, T., Carpentier, P., Sever, F., Terrien, M., Dobias, F., Juers, D. H., Flot, D., Mueller-Dieckmann, C., Leonard, G. A., De Sanctis, D. \& Royant, A. (2015). Acta Cryst. D71, 15-26.

Swartz, T. E., Corchnoy, S. B., Christie, J. M., Lewis, J. W., Szundi, I., Briggs, W. R. \& Bogomolni, R. A. (2001). J. Biol. Chem. 276, 36493-36500.

Theveneau, P., Baker, R., Barrett, R., Beteva, A., Bowler, M. W., Carpentier, P., Caserotto, H., Sanctis, D., Dobias, F., Flot, D., Guijarro, M., Giraud, T., Lentini, M., Leonard, G. A., Mattenet, M., McCarthy, A. A., McSweeney, S. M., Morawe, C., Nanao, M., Nurizzo, D., Ohlsson, S., Pernot, P., Popov, A. N., Round, A., Royant, A., Schmid, W., Snigirev, A., Surr, J. \& MuellerDieckmann, C. (2013). J. Phys. Conf. Ser. 425, 012001.

Wheeler, M. J., Russi, S., Bowler, M. G. \& Bowler, M. W. (2012). Acta Cryst. F68, 111-114.

Wöhri, A. B., Katona, G., Johansson, L. C., Fritz, E., Malmerberg, E., Andersson, M., Vincent, J., Eklund, M., Cammarata, M., Wulff, M., Davidsson, J., Groenhof, G. \& Neutze, R. (2010). Science, 328, 630-633. 